\title{
Phase-amplitude coupling between interictal high-frequency activity and slow waves in epilepsy surgery
}

\author{
Hirotaka Motoi ${ }^{1}$ | Makoto Miyakoshi ${ }^{2}$ | Taylor J. Abel ${ }^{3}$ | Jeong-Won Jeong ${ }^{1,4}$ | \\ Yasuo Nakai $^{1}$ | Ayaka Sugiura ${ }^{1}$ | Aimee F. Luat ${ }^{1,4}$ | Rajkumar Agarwal ${ }^{1,4}$ | \\ Sandeep Sood $^{5}$ | Eishi Asano ${ }^{1,4}$
}

${ }^{1}$ Department of Pediatrics, Children's Hospital of Michigan, Wayne State University, Detroit Medical Center, Detroit, Michigan

${ }^{2}$ Swartz Center for Computational Neuroscience, Institute for Neural Computation, University of California San Diego, La Jolla, California

${ }^{3}$ Division of Neurosurgery, Hospital for Sick Children, Toronto, Ontario, Canada

${ }^{4}$ Department of Neurology, Children's Hospital of Michigan, Wayne State University, Detroit Medical Center, Detroit, Michigan

${ }^{5}$ Department of Neurosurgery, Children's Hospital of Michigan, Wayne State University, Detroit Medical Center, Detroit, Michigan

Correspondence: Eishi Asano, Department of Neurodiagnostics, Children's Hospital of Michigan, Wayne State University, 3901 Beaubien St, Detroit, MI 48201

(easano@med.wayne.edu)

and

Makoto Miyakoshi, Swartz Center for Computational Neuroscience, University of California San Diego, 9500 Gilman

Drive, La Jolla, CA 92093-0559

(mmiyakoshi@ucsd.edu).

\section{Funding information}

National Institute of Neurological Disorders and Stroke, Grant/Award Number: NS047550, NS064033, NS089659

\begin{abstract}
Objective: We hypothesized that the modulation index (MI), a summary measure of the strength of phase-amplitude coupling between high-frequency activity $(>150 \mathrm{~Hz})$ and the phase of slow waves $(3-4 \mathrm{~Hz})$, would serve as a useful interictal biomarker for epilepsy presurgical evaluation.

Methods: We investigated 123 patients who underwent focal cortical resection following extraoperative electrocorticography recording and had at least 1 year of postoperative follow-up. We examined whether consideration of MI would improve the prediction of postoperative seizure outcome. MI was measured at each intracranial electrode site during interictal slow-wave sleep. We compared the accuracy of prediction of patients achieving International League Against Epilepsy class 1 outcome between the full multivariate logistic regression model incorporating $\mathrm{MI}$ in addition to conventional clinical, seizure onset zone (SOZ), and neuroimaging variables, and the reduced logistic regression model incorporating all variables other than MI.
\end{abstract}

Results: Ninety patients had class 1 outcome at the time of most recent follow-up (mean follow-up $=5.7$ years). The full model had a noteworthy outcome predictive ability, as reflected by regression model fit $R^{2}$ of 0.409 and area under the curve (AUC) of receiver operating characteristic plot of 0.838 . Incomplete resection of $\mathrm{SOZ}$ $(P<0.001)$, larger number of antiepileptic drugs at the time of surgery $(P=0.007)$, and larger MI in nonresected tissues relative to that in resected tissue $(P=0.020)$ were independently associated with a reduced probability of class 1 outcome. The reduced model had a lower predictive ability as reflected by $R^{2}$ of 0.266 and AUC of 0.767 . Anatomical variability in MI existed among nonepileptic electrode sites, defined as those unaffected by magnetic resonance imaging lesion, SOZ, or interictal spike discharges. With MI adjusted for anatomical variability, the full model yielded the outcome predictive ability of $R^{2}$ of 0.422 , AUC of 0.844 , and sensitivity/specificity of $0.86 / 0.76$.

Significance: MI during interictal recording may provide useful information for the prediction of postoperative seizure outcome.

\section{K E Y W O R D S}

epileptogenic zone, high-frequency oscillations, invasive recording, irritative zone, video-EEG monitoring 


\section{1 | INTRODUCTION}

The epileptogenic zone (EZ) is conceptually defined as the region, the resection of which is necessary and sufficient to achieve seizure freedom. ${ }^{1,2}$ Many investigators emphasize the value of ictal recording ${ }^{3-6}$ and suggest that the seizure onset zone (SOZ), defined as the region initiating ictal discharges during habitual seizures, is likely to comprise the EZ. ${ }^{2,7,8}$ As the sampling rate of digital recording systems have recently improved, investigators have described the value of interictal high-frequency activity at $>80 \mathrm{~Hz}$ $\left(\mathrm{HFA}_{>80 \mathrm{~Hz}}\right)$ for localizing the EZ. Specifically, resection of recording sites showing frequent episodes of HFA during invasive monitoring has been associated with excellent seizure outcome. ${ }^{9-16}$ However, whether preresection interictal HFA can inform postresection clinical outcome, independently of the effects of resection size, SOZ on ictal recording, and neuroimaging data, remains an open question. ${ }^{16}$

To evaluate whether interictal coupling between HFA and slow waves could be used as a preoperative electrographic biomarker, we examined whether a modulation index (MI) was associated with postoperative success rates in 123 patients with drug-resistant focal epilepsy. $\mathrm{MI}_{\text {(HFA \& slow waves) }}$ is a summary measure of the strength of coupling between HFA amplitude and slow-wave phase. ${ }^{17,18}$ Within the SOZ of drug-resistant focal epilepsy, it is typical for 3- to $4-\mathrm{Hz}$ slow waves to immediately follow interictal spike discharges associated with increased HFA. ${ }^{19-21}$ Our recent electrocorticographic (ECoG) study demonstrated that $\mathrm{MI}_{(>150 \mathrm{~Hz} \& 3-4 \mathrm{~Hz})}$ was positively correlated to the occurrence rate of $\mathrm{HFA}_{>150 \mathrm{~Hz}}$ at a given recording site during interictal slow-wave sleep and that the SOZ was predicted by $\mathrm{MI}_{(>150 \mathrm{~Hz}}$ \& $\left.3-4 \mathrm{~Hz}\right)$ equally with $\mathrm{MI}_{(>80 \mathrm{~Hz}}$ \& $\left.\quad 3-4 \mathrm{~Hz}\right)$ and $\mathrm{MI}_{(>250 \mathrm{~Hz}}$ \& $\left.3-4 \mathrm{~Hz}\right) \cdot{ }^{19}$ Taken together, measurement of $\mathrm{MI}_{(>150 \mathrm{~Hz} \& 3-4 \mathrm{~Hz})}$ was expected to effectively quantify the irritative zone, ${ }^{1}$ by delineating the severity and spatial gradient of interictal spike-andwave discharges, which are characterized by increased $\mathrm{HFA}_{>150 \mathrm{~Hz}}$ amplitude stereotypically coupled with a phase of slow waves $3-4 \mathrm{~Hz}$. The goal of this study was to test the hypothesis that the full multivariate logistic model also incorporating $\mathrm{MI}_{(>150 \mathrm{~Hz}}$ \& $\left.3-4 \mathrm{~Hz}\right)$ would more accurately predict patients achieving surgical success compared to the reduced model only incorporating the conventional clinical, $\mathrm{SOZ}$, and neuroimaging variables.

As an additional analysis, we determined whether anatomical variability in $\mathrm{MI}_{(>150 \mathrm{~Hz} \text { \& } 3-4 \mathrm{~Hz})}$ during slowwave sleep would exist among nonepileptic electrode sites, defined as those unaffected by magnetic resonance imaging (MRI) lesion, SOZ, or interictal spike discharges. $^{22}$ We believed this analysis was necessary for

\section{Key Points}

- Logistic regression model considering clinical, seizure onset zone, and imaging variables alone predicted postoperative seizure outcome

- The full model considering interictal modulation index in addition to the aforementioned variables better predicted seizure outcome

- Anatomical variability of interictal modulation index was suggested to exist across nonepileptic electrode sites

- Adjustment of interictal modulation index for anatomical variability minimally improved the outcome predictive ability of the full model

- The full model incorporating adjusted modulation index had sensitivity/specificity of $0.86 / 0.76$ for predicting seizure outcome

validating the application of $\mathrm{MI}_{(>150 \mathrm{~Hz} \& 3-4 \mathrm{~Hz})}$ in presurgical evaluation, because previous studies of nonepileptic eloquent cortex reported that the degree of phase-amplitude coupling between HFA and theta/alpha/beta activity was modulated by sensorimotor or cognitive tasks during wakefulness. ${ }^{17,23}$ If our analysis suggested that nonepileptic $\mathrm{MI}_{(>150 \mathrm{~Hz}}$ \& $\left.3-4 \mathrm{~Hz}\right)$ varies across regions of interest (ROIs) during slow-wave sleep, our subsequent analysis determined whether the outcome prediction with the full multivariate logistic regression model would be further improved by incorporating $\left.\mathrm{MI}_{(>150 \mathrm{~Hz}} \& \quad 3-4 \mathrm{~Hz}\right)$ adjusted for such anatomical variability. Finally, we determined the effect of sleep staging on the $\mathrm{MI}_{(>150 \mathrm{~Hz} \& 3-4 \mathrm{~Hz})}$ at $\mathrm{SOZ}$, to confirm the validity of usage of slow-wave sleep ECoG data in presurgical evaluation.

\section{2 | MATERIALS AND METHODS}

\section{1 | Patients}

The inclusion criteria were focal resective surgery following extraoperative ECoG recording with a sampling rate of $1000 \mathrm{~Hz}$ at Children's Hospital of Michigan or Harper University Hospital in Detroit between January 2007 and October 2016. Patients were excluded from the study if: (1) the EZ was determined to be present independently in both hemispheres on the basis of the noninvasive evaluation, ${ }^{24}$ (2) they needed hemispherotomy or hemispherectomy, (3) extensive brain malformations prevented consistent analysis of major anatomical landmarks, ${ }^{25}$ (4) postoperative follow-up was $<1$ year, (5) the patient had prior resective epilepsy surgery, and (6) the patient's age 
TABLE 1 Patient profiles

\begin{tabular}{ll} 
Total number of patients & $\mathbf{1 2 3}$ \\
Mean age, y & 13.4 \\
\hline Age range, y & $4-44$ \\
\hline Female patients & $48.8 \%$ \\
\hline Patients with daily seizures & $35.0 \%$ \\
\hline 1 AED & $30.1 \%$ \\
\hline 2 AEDs & $43.9 \%$ \\
\hline 3 AEDs & $25.2 \%$ \\
\hline 4 AEDs & $0.0 \%$ \\
\hline 5 AEDs & $0.8 \%$ \\
\hline Left-hemispheric focus & $54.5 \%$ \\
\hline Cortical lesion on MRI & \\
\hline Habitual seizure events captured & $58.0 \%$ \\
\hline during extraoperative ECoG & $89.4 \%$ \\
\hline Incomplete resection of SOZ & \\
\hline Extratemporal lobe resection & $13.80 \%$ \\
\hline Mean of resection size & $60.2 \%$ \\
\hline Range of resection size & $15.30 \%$ \\
\hline Class 1 outcome & $0.6 \%-91.6 \%$ \\
\hline Class 2-6 outcomes & $73.20 \%$ \\
& $(90.80 \%$ \\
\hline
\end{tabular}

AED, antiepileptic drugs; ECoG, electrocorticography; MRI, magnetic resonance imaging; SOZ, seizure onset zone.

${ }^{\text {a }}$ Thirty-seven patients showed brain malformations (including focal cortical dysplasia, cortical tuber, and ulegyria) on MRI, 25 showed tumor, five showed medial-temporal sclerosis, four showed gliotic changes associated with atrophy, one showed arteriovenous malformation.

b Including three patients achieving class 1 outcome following the second surgery and three patients failing to achieve class 1 outcome following the initial and second surgeries.

was $<4$ years. ${ }^{26}$ We studied a consecutive series of 123 patients (Table 1) who satisfied the aforementioned criteria. This study protocol was approved by the institutional review board at Wayne State University, and written informed consent was obtained from each patient or the guardian of each pediatric patient.

\section{2 | Electrocorticography}

The principal methods of ECoG data acquisition are identical to those previously described. ${ }^{25,27}$ Platinum subdural disk electrodes (10 mm center-to-center distance) were placed on the epileptic hemisphere. Electrode placement was guided by semiology, scalp video-electroencephalography, and neuroimaging data. Before dural closure, intraoperative photographs of the brain and subdural electrodes were taken. Following closure, surface electromyographic electrodes were placed on the deltoid muscles ${ }^{28}$ and electrooculographic electrodes were placed $2.5 \mathrm{~cm}$ below and $2.5 \mathrm{~cm}$ lateral to the outer canthi. ${ }^{29}$

Extraoperative ECoG signals were recorded with a band pass of $0.016-300 \mathrm{~Hz}$ for 3-7 days. The averaged voltage of ECoG signals from the fifth to sixth intracranial electrodes of the amplifier was used as the original reference, and signals were remontaged to a common average reference. Antiepileptic drugs (AEDs) were discontinued and resumed once $\mathrm{SOZ}$ was determined. The $\mathrm{SOZ}$ is defined as electrode sites initially showing sustained rhythmic ECoG changes prior to the onset of habitual clinical seizure symptoms, not explained by state changes, and clearly distinguished from interictal activity. ${ }^{8}$ Ictal patterns of epileptic spasms are characterized by fast-wave bursts quickly propagated to multiple lobes, ${ }^{28}$ whereas those of focal seizures are characterized by repetitive spike-and-wave discharges or focal fast-wave discharges followed by gradual propagation to the surrounding regions. ${ }^{8}$ Electrode sites affected by artifacts were excluded from further analysis. A total of 12964 electrodes (mean $=105.4$ electrodes per a patient, range $=32-152$ per patient) were included into the multivariate logistic regression analyses.

\section{3 | Magnetic resonance imaging}

Preoperative MRI was reviewed by an experienced pediatric neuroradiologist who was blinded to scalp video-electroencephalographic data. ${ }^{24} \mathrm{~A}$ three-dimensional surface image was created with the locations of electrodes coregistered on it. ${ }^{25}$ The spatial normalization of electrode sites was performed using FreeSurfer scripts (http://surfer.nmr. mgh.harvard.edu). All electrode sites on each subject's FreeSurfer brain surface were plotted on the averaged FreeSurfer surface image. ${ }^{25,26}$ Parcellation of cortical ROIs was performed at both individual and spatially normalized brain surfaces (Figure 1).

\subsection{Surgical decision making}

Surgical resection was guided by the clinical factors, semiology, visual assessment of extraoperative ECoG, extent of lesion, and eloquent areas. ${ }^{8} \mathrm{We}$ intended to completely remove SOZ, sites of frequent interictal spikes nonattributable to propagation from $\mathrm{SOZ}$, and lesions surrounding $\mathrm{SOZ}$ (if present). When the $\mathrm{SOZ}$ was not identified, we planned to remove sites showing frequent interictal spikewave discharges and the associated lesion. Simultaneously, we intended to preserve the eloquent areas and their associated vascular structures along sulcal boundaries. In cases where eloquent cortex overlapped with the regions presumed to be epileptogenic, the exact resection margin was determined, on an individualized basis, after intense discussion with the family of a given patient. 

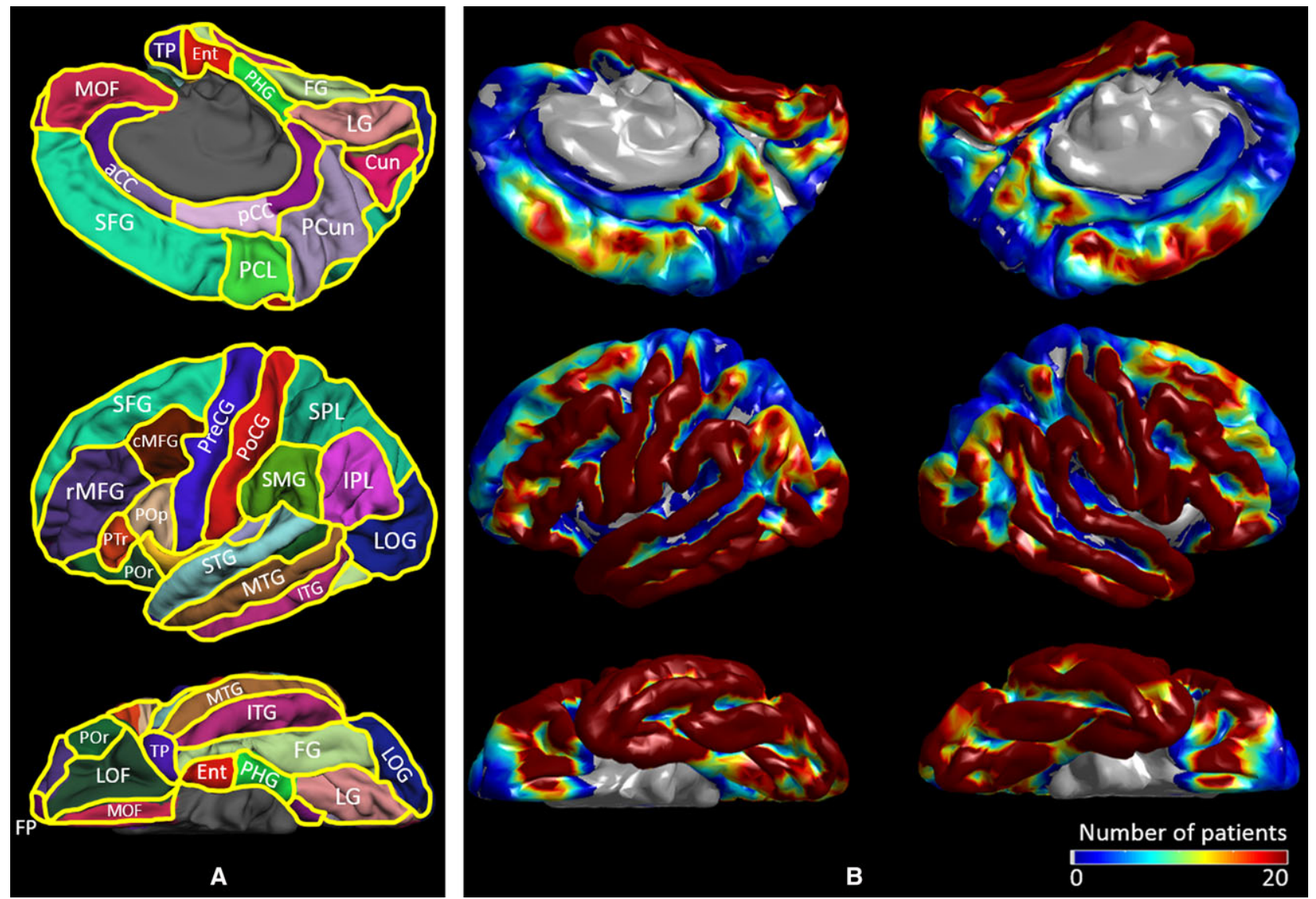

FIGURE 1 Regions of interest (ROIs) and spatial distribution of intracranial electrode coverage. A, The locations of 28 ROIs are indicated. aCC, anterior cingulate cortex; cMFG, caudal middle frontal gyrus; Cun, cuneus gyrus; Ent, entorhinal gyrus; FG, fusiform gyrus; FP, frontal pole; IPL, inferior parietal lobule; ITG, inferior temporal gyrus; LG, lingual gyrus; LOF, lateral orbitofrontal gyrus; LOG, lateral occipital gyrus; MOF, medial orbitofrontal gyrus; MTG, middle temporal gyrus; pCC, posterior cingulate cortex; PCL, paracentral lobule; PCun, precuneus gyrus; PHG, parahippocampal gyrus; PoCG, postcentral gyrus; POp/POr/PTr, pars opercularis/pars triangularis/pars orbitalis within the inferior frontal gyrus; PreCG, precentral gyrus; rMFG, rostral middle frontal gyrus; SFG, superior frontal gyrus; SMG, supramarginal gyrus; SPL, superior parietal lobule; STG, superior temporal gyrus; TP, temporal pole. B, The spatial distribution of electrode coverage is indicated (123 patients; 12964 electrode sites) [Color figure can be viewed at wileyonlinelibrary.com]

\subsection{Measurement of the size of resection}

Intraoperative photographs were obtained prior to dural closure to confirm the extent of resection. We determined whether all electrode sites marked as SOZ were completely removed. In cases where SOZ sites were located at the top of a sulcus and resection was completed up to that sulcus, the SOZ was considered to be completely removed. The extent of resection (percentage of the affected hemisphere) was quantified using FreeSurfer scripts by H.M. while blinded to the postoperative seizure outcome.

\section{6 | Modulation index}

As this is a retrospective observational study, the interictal $\left.\mathrm{MI}_{(>150 \mathrm{~Hz}} \& 3-4 \mathrm{~Hz}\right)$ did not affect our surgical decision making. Measurement of the MI was performed using EEGLAB Toolbox winPACT (https://sccn.ucsd.edu/wiki/
WinPACT). $\mathrm{MI}_{(>150 \mathrm{~Hz} \text { \& } 3-4 \mathrm{~Hz})}$ during slow-wave sleep was calculated at each electrode site using the algorithm identical to that previously reported. ${ }^{17,18}$ Ten earliest available, 30-second, least artifactual epochs of slow-wave sleep $^{30}$ were selected from the first (or the second if needed) evening of extraoperative $\mathrm{ECoG}$ recording by H.M. while blinded to the seizure outcome. All selected epochs were $\geq 2$ hours apart from seizure events, and highpass filtered at $150 \mathrm{~Hz}$. All ECoG data points were Hilberttransformed and used for computing $\mathrm{MI}_{(>150 \mathrm{~Hz} \& 3-4 \mathrm{~Hz})}$, the strength of coupling between the amplitude of $\mathrm{HFA}_{>150 \mathrm{~Hz}}$ and the instantaneous phase of local slow waves $3-4 \mathrm{~Hz}$. Each electrode site was finally assigned an $\mathrm{MI}_{(>150 \mathrm{~Hz}}$ \& $\left.3-4 \mathrm{~Hz}\right)$ value averaged across ten 30 -second epochs.

Our MI algorithm does not differentiate between HFA derived from oscillations and that from nonoscillatory spike discharges. ${ }^{31,32}$ We believe our analytic approach is justified in this clinical study, as oscillatory and nonoscillatory 
HFAs are both reported to be useful to localize SOZ. ${ }^{33}$ Some reported that interictal $\mathrm{HFA}_{>200 \mathrm{~Hz}}$ on clinical electrodes is mostly derived from nonoscillatory spike discharges. ${ }^{32}$ Others reported that $\mathrm{HFA}_{>250 \mathrm{~Hz}}$ disentangled from nonoscillatory spikes had insufficient sensitivity to localize the SOZ. ${ }^{34}$ Figure 2 helps readers in comprehending the relationship between representative ECoG traces and resulting $\mathrm{MI}_{(>150 \mathrm{~Hz} \& 3-4 \mathrm{~Hz})}$ values. Nonoscillatory spikes are certainly expected to contribute to increased $\mathrm{HFA}_{>150 \mathrm{~Hz}}$ as well as increased $\mathrm{MI}_{(>150 \mathrm{~Hz} \& 3-4 \mathrm{~Hz})} \cdot{ }^{35} \mathrm{Fig}-$ ure $\mathrm{S} 1$ presents the distribution of $\mathrm{MI}_{(>150 \mathrm{~Hz}}$ \& $\left.3-4 \mathrm{~Hz}\right)$ in (1) SOZ, (2) spiking sites (non-SOZ but affected by interictal spike discharges), and (3) nonepileptic sites.

\section{7 | Seizure outcome}

After a minimum of 1 year, postoperative seizure outcome was classified according to the International League Against Epilepsy classification. ${ }^{36}$ Medical charts and telephone calls were used by investigators unaware of the results of MI analysis. Class 1 outcome was treated as success and others as failure. Requirement of reoperation was treated as failure, even if patients achieved class 1 outcome following the second surgery.

\section{8 | Outcome prediction using multivariate logistic regression models}

The statistical analysis was performed using SPSS Statistics v25 (IBM, Armonk, New York). The significance was set at $P<0.05$. We initially determined how well patients achieving surgical success would be predicted by a multivariate logistic regression model incorporating clinical, $\mathrm{SOZ}$, and neuroimaging variables, but not an MI variable (referred to as "reduced model"). The outcome measure was achievement of International League Against Epilepsy class 1 outcome. The predictor variables (Table 2) included: age, gender, presence of daily seizures, number

\section{ECoG traces \\ (HFF: $300 \mathrm{~Hz}$; TC: $2 \mathrm{~s}$ ) \\ Zoomed traces (HFF: $300 \mathrm{~Hz}$; TC: $0.001 \mathrm{~s}$ )}
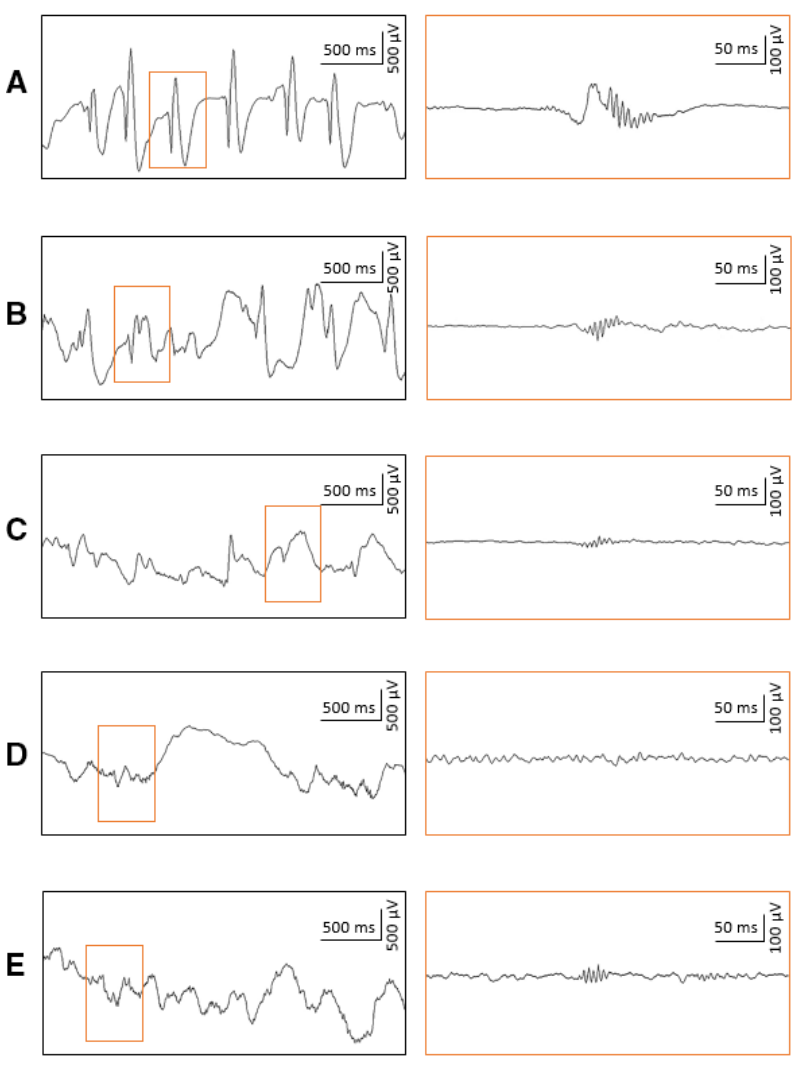
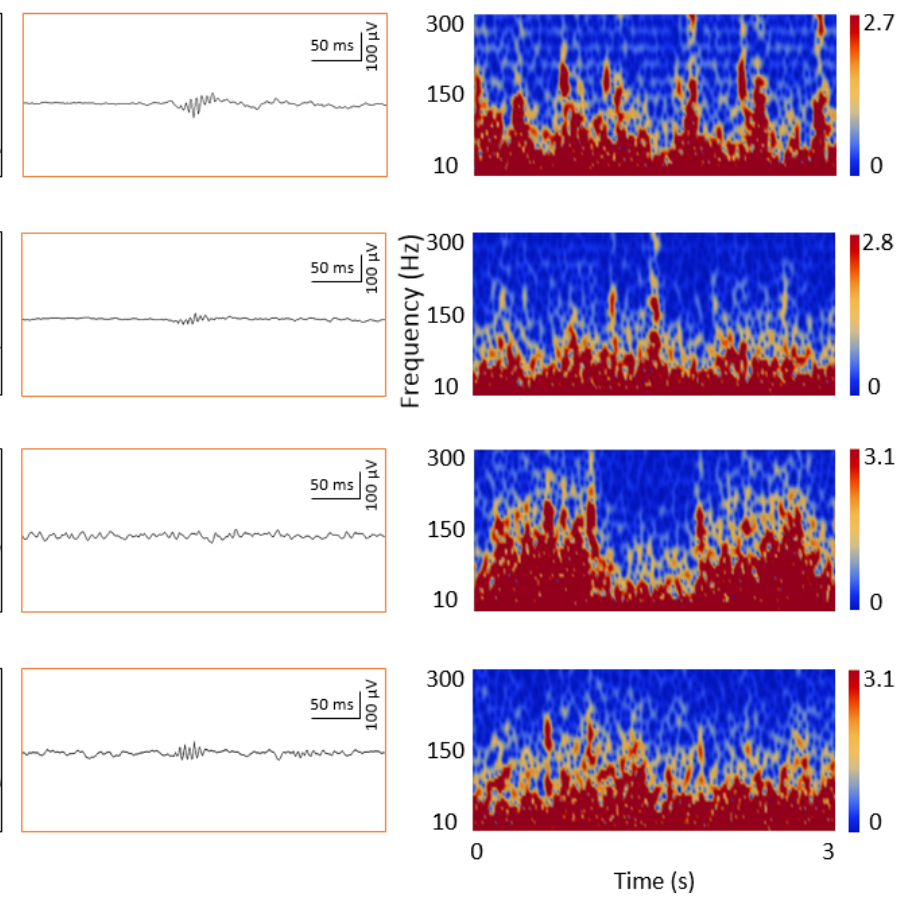

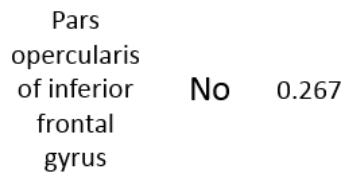

Cuneus
gyrus

No

0.198

Precentral gyrus

No

0.078

opercularis
of inferior Yes 0.720
frontal
gyrus

Caudal
middle
frontal
gyrus

(2)

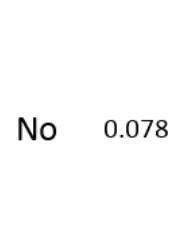

F I G URE 2 Relationship between electrocorticographic (ECoG) trace and modulation index (MI) in a 7-year-old boy with frontal lobe epilepsy. Representative ECoG waveforms are presented with their corresponding locations, time-frequency plots, and $\mathrm{MI}_{(>150 \mathrm{~Hz}}$ \& $\left.3-4 \mathrm{~Hz}\right)$ values. A, B, Classified as seizure onset zone (SOZ). The spectral features seen on time-frequency plots are consistent with the notion that observed high-frequency activity $>150 \mathrm{~Hz}$ was mostly derived from nonoscillatory spikes. ${ }^{31-33,35} \mathrm{C}$, Classified as a site showing interictal spike discharges. D, E, Classified as nonepileptic sites. ROI, region of interest; TC, time constant [Color figure can be viewed at wileyonlinelibrary.com] 
of oral AEDs taken immediately prior to intracranial electrode placement, affected hemisphere, cortical lesion on MRI, habitual clinical seizure events captured during extraoperative ECoG recording, incomplete resection of SOZ, necessity of extratemporal lobe resection, and size of resection. This analysis yielded a model fit $R^{2}$ (ranging from 0 to 1), indicating how much the seizure outcome can be explained by the variance of collective variables incorporated in the model. Each patient was given a predicted probability of surgical success based on her/his clinical, SOZ, and neuroimaging profiles. Receiver operating characteristic (ROC) analysis, ${ }^{8,19,34}$ applied to the predicted probabilities assigned to all 123 patients, determined the accuracy of the reduced model in predicting surgical success. The area under the curve (AUC) of a given ROC plot (Figure 3) indicates the overall power of outcome prediction $(0.5=$ random prediction, $1.0=$ perfect prediction $)$.

We subsequently determined whether a multivariate logistic regression model incorporating an MI variable in addition to the aforementioned clinical, SOZ, and neuroimaging variables (referred to as "full model") would improve the outcome predictive ability. The added MI predictor variable was subtraction $M I$, defined as subtraction of $\mathrm{MI}_{(>150 \mathrm{~Hz}}$ \& $\left.3-4 \mathrm{~Hz}\right)$ averaged across all preserved sites from $\mathrm{MI}_{(>150 \mathrm{~Hz}}$ \& $\left.3-4 \mathrm{~Hz}\right)$ averaged across all resected sites. A patient would be assigned a larger subtraction MI, if areas showing larger $\mathrm{MI}_{(>150 \mathrm{~Hz}}$ \& $\left.3-4 \mathrm{~Hz}\right)$ were resected and those showing relatively smaller $\mathrm{MI}_{(>150 \mathrm{~Hz}}$ \& $\left.3-4 \mathrm{~Hz}\right)$ were preserved. Assessment of $R^{2}$ and AUC on ROC plot effectively allowed us to estimate how much more accurately the full model, compared to the reduced model, predicted surgical success. Comparison of 122 sensitivity $\times$ specificity values consisting of each ROC plot determined whether the size of AUC differed between two models (studentized bootstrap statistics).

\section{9 | Variability in MI across anatomical regions and sleep stages}

We determined the spatial characteristics of $\left.\mathrm{MI}_{(>150 \mathrm{~Hz}} \& 3-4 \mathrm{~Hz}\right)$ at nonepileptic electrode sites during slow-wave sleep as well as the effect of sleep staging on the overall $\mathrm{MI}_{(>150 \mathrm{~Hz}}$ \& $\left.3-4 \mathrm{~Hz}\right)$ profiles. These additional analyses were applied to 47 patients (age range $=4-19$ years) in whom ECoG signals were sampled from all four lobes, sleep spindles were visualized at nonepileptic frontal regions, and posterior dominant alpha activity was noted at nonepileptic occipital regions during wakefulness. Such widespread electrode coverage, together with electrooculography/electromyography, allowed accurate sleep staging and measurement of $\mathrm{MI}_{(>150 \mathrm{~Hz}}$ \& 3-4 Hz) during wakefulness, sleep stage 1 , sleep stage 2 , and rapid eye movement (REM) sleep. ${ }^{30}$
To determine whether interictal $\mathrm{MI}_{(>150 \mathrm{~Hz}}$ \& $\left.3-4 \mathrm{~Hz}\right)$ within an ROI differed from that within the rest of the regions, the mixed model analysis ${ }^{37}$ was applied to 2477 nonepileptic electrode sites in the 47 patients. The dependent variable was $\mathrm{MI}_{(>150 \mathrm{~Hz} \text { \& } 3-4 \mathrm{~Hz})}$ during slow-wave sleep. The following covariates were treated as fixed effects: age, gender, presence of daily seizures, number of AEDs, affected hemisphere, lesion on MRI, habitual clinical seizure events during extraoperative $\mathrm{ECoG}$, and one of the 28 ROIs listed in Figure 1. "Intercept" and "patient" were treated as random effects. Bonferroni correction was employed for multiple comparisons across 28 ROIs.

To determine the effect of sleep stages on $\mathrm{MI}_{(>150 \mathrm{~Hz}}$ \& 3-4 Hz), another mixed model analysis was subsequently applied to 4996 sites (2477 nonepileptic sites, 440 SOZ sites, and 2079 spiking but non-SOZ sites) in the same cohort of 47 patients. The dependent variable was $\mathrm{MI}_{(>150 \mathrm{~Hz}}$ \& 3-4 Hz). The following covariates were treated as fixed effects: age, gender, presence of daily seizures, number of AEDs, affected hemisphere, lesion on MRI, habitual clinical seizure events captured during extraoperative ECoG, SOZ, presence of interictal spike discharges, sleep or wakefulness, non-REM sleep, and slow-wave sleep.

\subsection{0 | Full multivariate logistic regression model incorporating MI adjusted for anatomical locations}

In case the aforementioned mixed model analysis on 47 patients revealed that $\mathrm{MI}_{(>150 \mathrm{~Hz} \& 3-4 \mathrm{~Hz})}$ at nonepileptic electrode sites within an ROI differed from that within the rest of the ROIs, we modified the full multivariate logistic regression model by incorporating $\left.\mathrm{MI}_{(>150 \mathrm{~Hz}} \& 3-4 \mathrm{~Hz}\right)$ adjusted for the anatomical location (ie, $\mathrm{aMI}_{[>150 \mathrm{~Hz} \& 3-4 \mathrm{~Hz}]}$ ). Specifically, $\mathrm{MI}_{(>150 \mathrm{~Hz} \& 3-4 \mathrm{~Hz})}$ was adjusted by its mixed model estimate, if a given electrode site was within one of the ROIs showing a significant difference. Using collective data from all 123 patients, we finally determined whether the outcome predictive ability would be further improved by the full model incorporating subtraction $a M I$, defined as subtraction of $\left.\mathrm{aMI}_{(>150 \mathrm{~Hz}} \& 3-4 \mathrm{~Hz}\right)$ averaged across all preserved sites from $\operatorname{aMI}_{(>150 \mathrm{~Hz} \& 3-4 \mathrm{~Hz})}$ averaged across all resected sites.

\section{3 | RESULTS}

\section{1 | Outcome prediction}

International League Against Epilepsy class 1, 2, 3, 4, 5, and 6 outcomes were noted in 90, 3, 13, 9, 8, and 0 patients, respectively (mean follow-up period $=5.7$ years, range $=1.0-10.8$ years). The reduced model had a 
TABLE 2 Results of multivariate logistic regression analysis

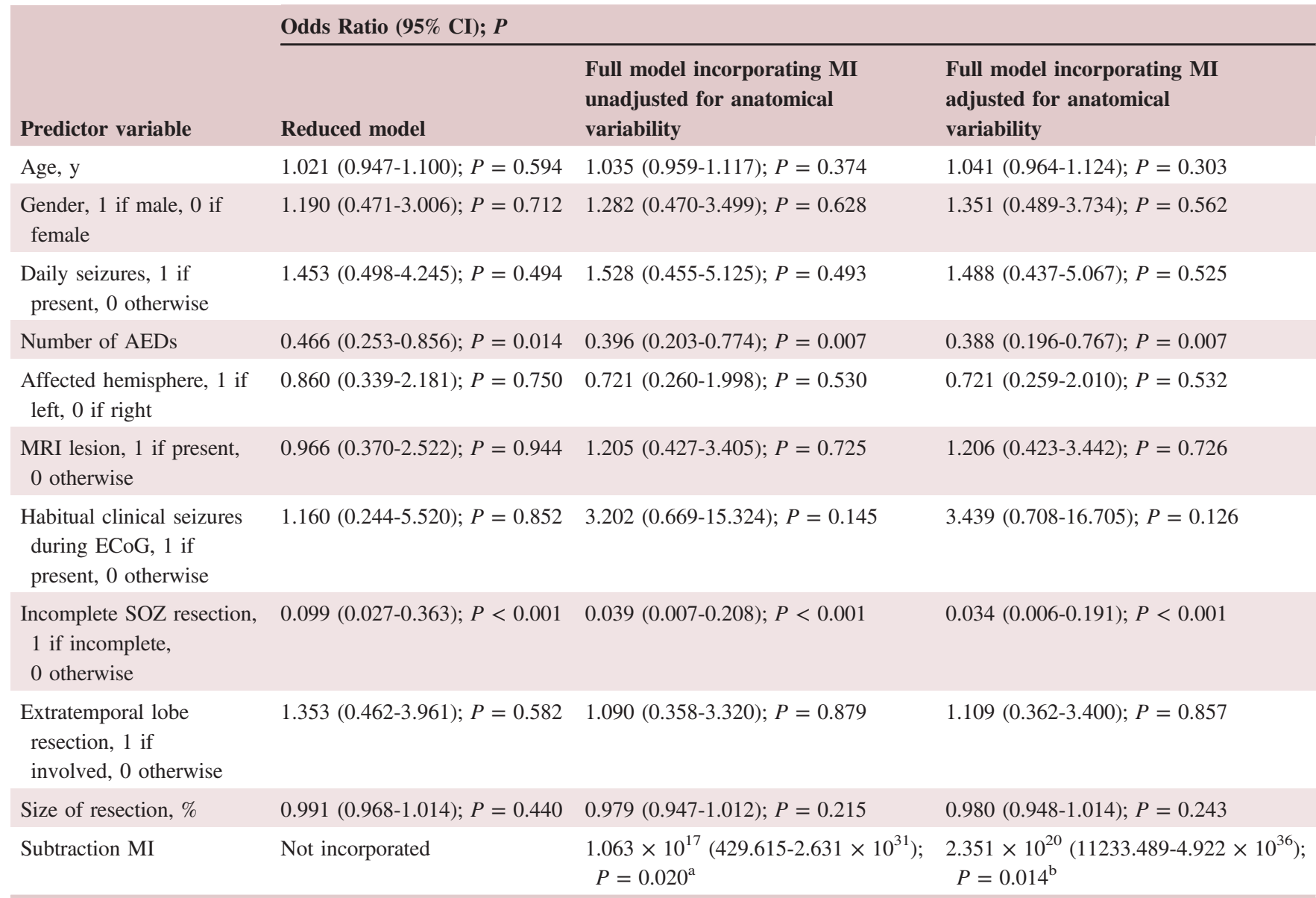

Subtraction MI is defined as subtraction of MI averaged across all preserved sites from MI averaged across all resected sites. Only five of the 17 patients whose SOZ was incompletely removed achieved class 1 outcome, whereas 85 of the remaining 106 patients achieved such surgical success.

AED, antiepileptic drug; CI, confidence interval; ECoG, electrocorticography; MI, modulation index; MRI, magnetic resonance imaging; SOZ, seizure onset zone.

${ }^{a}$ In other words, each increase of 0.01 point increased the odds of surgical success by $48.0 \%$ (95\% CI $=6.3 \%-206.2 \%, P=0.020$ ).

${ }^{\mathrm{b}}$ Each increase of 0.01 point increased the odds of surgical success by $59.9 \%(95 \% \mathrm{CI}=9.8 \%-232.8 \%, P=0.014)$.

significant outcome predictive ability $\left(R^{2}=0.266\right.$, $P=0.006$ ). Incomplete $\mathrm{SOZ}$ resection (odds ratio $[\mathrm{OR}]=$ $0.099, P<0.001)$ and larger number of AEDs (OR $=$ $0.466, P=0.014)$ were independently associated with a smaller chance of success (Table 2). The accuracy of outcome prediction rated by ROC analysis was AUC of 0.767 $(P<0.001)$. When the sensitivity was set to 0.86 , the specificity was 0.48 (Figure 3).

The full model, which incorporated subtraction MI, improved the outcome predictive ability $\left(R^{2}=0.409\right.$, $P<0.001)$. Incomplete $\mathrm{SOZ}$ resection $(\mathrm{OR}=0.039$, $P<0.001)$ and larger number of AEDs (OR $=0.396$, $P=0.007)$ were associated with a smaller chance of success. Larger subtraction MI was independently associated with a greater chance of success (Table 2), and each increase of 0.01 point increased the odds of surgical success by $48 \%(P=0.020)$. The accuracy of outcome prediction rated by ROC analysis was AUC of 0.838 and sensitivity/specificity of $0.86 / 0.73(P<0.001$; Figure 3$)$. Studentized bootstrap statistics suggested that the full model had a larger AUC compared to the reduced model $(t=11.826, P<0.001)$.

\subsection{Anatomical variability in MI}

The mixed model analysis applied to 2477 nonepileptic electrode sites demonstrated the existence of anatomical variability in $\mathrm{MI}_{(>150 \mathrm{~Hz}}$ \& 3-4 Hz) during slow-wave sleep. The following six ROIs showed a difference in $\mathrm{MI}_{(>150 \mathrm{~Hz}}$ \& $3-4 \mathrm{~Hz})$ compared to the rest of the ROIs $(P<0.001)$. Lingual (estimate $=+0.016$ ), cuneus (estimate $=+0.018$ ), lateral-occipital (estimate $=+0.016$ ), and fusiform regions (estimate $=+0.004)$ had larger $\mathrm{MI}_{(>150 \mathrm{~Hz}}$ \& $\left.3-4 \mathrm{~Hz}\right)$ (Figure 4). Superior-temporal $\quad$ (estimate $=-0.004)$ and 


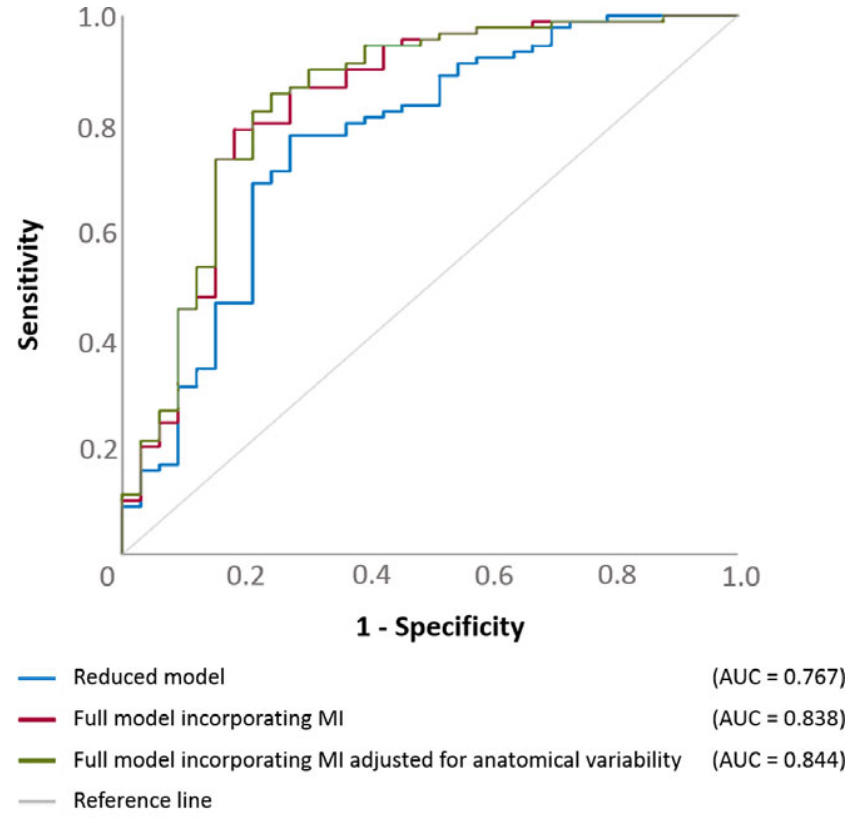

F I G URE 3 Receiver operating characteristic (ROC) plots. ROC plots indicate the model performance in predicting surgical success, defined as achievement of International League Against Epilepsy class 1 outcome. Blue line, the reduced logistic regression model incorporating clinical, seizure onset zone, and neuroimaging variables alone; red line, the full model also incorporating subtraction modulation index (MI; unadjusted for anatomical variability); green line, the full model also incorporating subtraction aMI (MI adjusted for anatomical variability). AUC, area under the curve [Color figure can be viewed at wileyonlinelibrary.com]

superior-frontal regions (estimate $=-0.005$ ) had smaller $\mathrm{MI}_{(>150 \mathrm{~Hz} \& 3-4 \mathrm{~Hz})}$.

\section{3 | Outcome prediction with MI adjusted for anatomical variation}

Because the anatomical variability in $\mathrm{MI}_{(>150 \mathrm{~Hz} \& 3-4 \mathrm{~Hz})}$ was suggested to exist among nonepileptic electrode sites, we decided to determine the outcome predictive ability of the full model incorporating $\left.\mathrm{aMI}_{(>150 \mathrm{~Hz}} \& 3-4 \mathrm{~Hz}\right)$. At a lateraloccipital site, for example, $\mathrm{aMI}_{(>150 \mathrm{~Hz} \& 3-4 \mathrm{~Hz})}$ was smaller by 0.016 than the original/unadjusted $\mathrm{MI}_{(>150 \mathrm{~Hz}}$ \& $\left.3-4 \mathrm{~Hz}\right)$. Conversely, $\mathrm{aMI}_{(>150 \mathrm{~Hz} \& 3-4 \mathrm{~Hz})}$ was treated as identical to $\left.\mathrm{MI}_{(>150 \mathrm{~Hz}} \& 3-4 \mathrm{~Hz}\right)$ in regions other than the aforementioned six ROIs. The modified full model had an $R^{2}$ of 0.422 $(P<0.001)$. Incomplete $\mathrm{SOZ}$ resection $(\mathrm{OR}=0.034$, $P<0.001)$ and larger number of AEDs $(\mathrm{OR}=0.388$, $P=0.007)$ were associated with a smaller chance of success. Larger subtraction aMI was independently associated with a greater chance of success (Table 2); thereby, each increase of 0.01 point increased the odds of surgical success by $59.9 \%(P=0.014)$; the accuracy of outcome prediction of the modified full model was rated as AUC of 0.844 and sensitivity/specificity of $0.86 / 0.76(P<0.001$; Figure 3).

\subsection{Effect of sleep stages on MI}

The spatial characteristics of $\mathrm{MI}_{(>150 \mathrm{~Hz}}$ \& 3-4 Hz) during each sleep stage are presented in Figure 4 . The mixed model analysis demonstrated that SOZ had greater $\mathrm{MI}_{(>150 \mathrm{~Hz} \& 3-4 \mathrm{~Hz})}$ compared to non-SOZ (estimate $=+0.045, t=+25.966, P<$ $0.001)$, and non-REM sleep had greater $\mathrm{MI}_{(>150 \mathrm{~Hz} \& 3-4 \mathrm{~Hz})}$ compared to the other stages (ie, wakefulness or REM sleep; estimate $=+0.004, t=+3.149, P=0.002)$. No significant difference in $\mathrm{MI}_{(>150 \mathrm{~Hz}}$ \& 3-4 Hz) was found between slowwave sleep and other states.

\section{4 | DISCUSSION}

\section{1 | Value of the irritative zone}

This study provides evidence that $\mathrm{MI}_{(>150 \mathrm{~Hz} \text { \& } 3-4 \mathrm{~Hz})}$ may be a useful adjunct for localization of the EZ. Incorporation of MI, regardless of the usage of indices adjusted or unadjusted for anatomical variability, improved seizure outcome prediction. Specifically, the outcome predictive ability rated by AUC of the ROC plot was improved from 0.767 to 0.838 (when unadjusted MI was used) or to 0.844 (when adjusted MI was used). One of the strengths of our study is its sample size, allowing assessment of the independent effects of multiple covariates including clinical, ictal ECoG, and neuroimaging variables. Our results support the theoretical notion that the EZ is optimally delineated by considering the spatial extents of SOZ and irritative zone., Based on their effect sizes (Table 2), we speculate that ictal recordings, compared to the interictal MI, provide more direct information to delineate the regions responsible for generation of habitual seizures.

\section{2 | Significance of anatomical variability in MI}

We observed anatomical variability in nonepileptic MI during slow-wave sleep. Specifically, occipital and fusiform regions showed relatively greater MI. Relative increase of MI in nonepileptic visual pathways is likely to be partly attributable to physiological augmentation of HFA taking place preferentially at a rate of $0.5-1 \mathrm{~Hz}$ during slow-wave sleep. ${ }^{19}$ Such augmentation of HFA, preferentially locked to the trough of slow waves $0.5-1 \mathrm{~Hz}$ during slow-wave sleep (Figure 2D), likely reflects increased cellular/synaptic activity (also known as up-states) that may play a role in low-order visual memory consolidation. ${ }^{29,38}$ In turn, each attenuation taking place between HFA augmentations likely reflects hyperpolarization and cellular silence (also known 


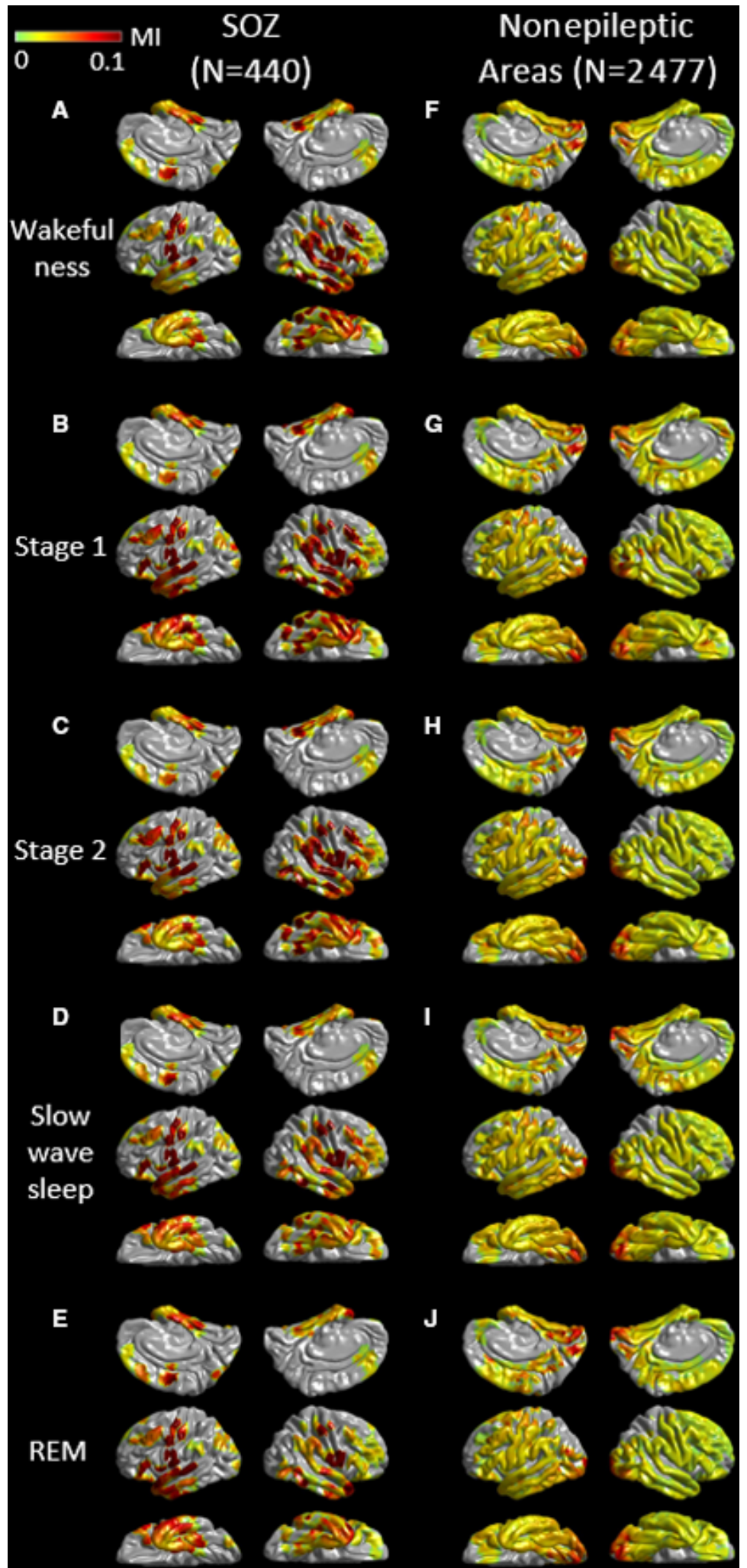

F I G URE 4 Modulation index during different sleep stages. The spatial characteristics of modulation index $(\mathrm{MI})_{(>150 \mathrm{~Hz} \& 3-4 \mathrm{~Hz})}$ during different sleep stages are presented. A-E, $\mathrm{MI}_{(>150 \mathrm{~Hz} \& 3-4 \mathrm{~Hz})}$ at 440 seizure onset zone (SOZ) electrode sites. F-J, $\mathrm{MI}_{(>150 \mathrm{~Hz} \& 3-4 \mathrm{~Hz})}$ at 2477 nonepileptic electrode sites, defined as those unaffected by magnetic resonance imaging lesion, SOZ, or interictal spike discharges. ${ }^{22} \mathrm{~K}$, The spatial distribution of electrode coverage of 47 patients used for this analysis. REM, rapid eye movement 


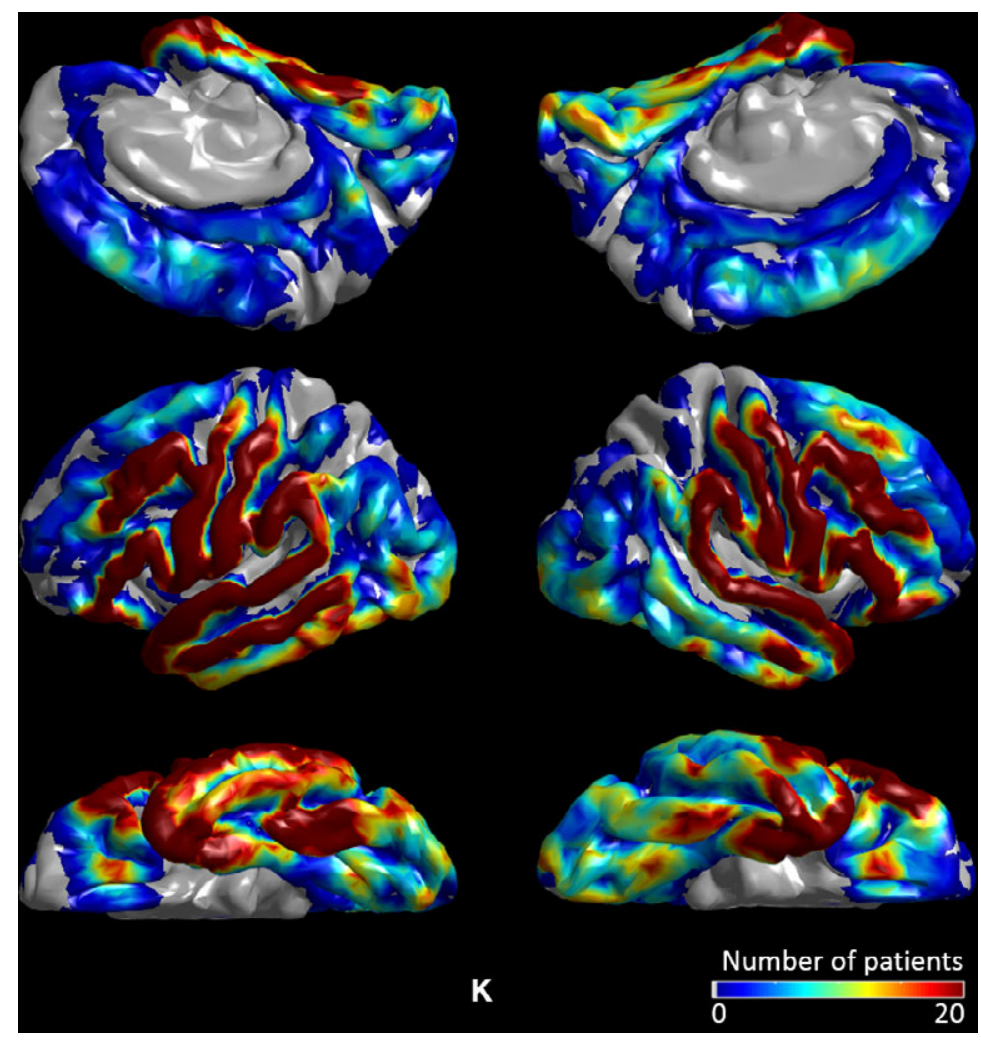

FIGURE 4 Continued.

as down-states). Superior-temporal and superior-frontal sites showed relatively smaller nonepileptic MI, but the magnitude of difference in these regions was much smaller compared to that in the occipital regions.

Because the existence of anatomical variability in nonepileptic MI has been suggested, we examined how well adjustment of MI for regional variability would further improve the performance of outcome prediction with the full model. This additional analysis demonstrated that incorporation of adjusted $\mathrm{MI}$ only resulted in a minimal improvement of outcome predictive ability $(\mathrm{AUC}=0.838$ $\rightarrow 0.844$; sensitivity/specificity $=0.86 / 0.73 \rightarrow 0.86 / 0.76$ ). It is possible that the ROIs used in this study may have been too large for optimal adjustment and that MI adjusted using smaller ROIs would have more effectively improved the accuracy of outcome prediction of the full model.

\section{3 | Methodological considerations}

The sample size, data availability by the time of surgery, and collinearity problems among the predictor variables need to be taken into account for the selection of predictor variables to be incorporated in the full multivariate logistic regression models. Presence/absence of cortical lesions on MRI was selected because MRI-lesional epilepsy, compared to nonlesional, has been reported to have a better postoperative seizure prognosis. ${ }^{39,40}$ Necessity of extratemporal lobe resection was also selected because such surgery is generally associated with a less favorable prognosis compared to resection confined to the temporal lobe. ${ }^{40}$ Neither pathological diagnosis nor postoperative MRI was incorporated in our models, as such data are available only after resective surgery. Individual three-dimensional surface images and intraoperative photographs allowed us to measure the size of resection prior to completion of the resective surgery. The duration of epilepsy was not incorporated in our regression models, because of the high collinearity between patient age and epilepsy duration. A larger number of oral AEDs was found to be associated with a smaller chance of success. An interpretation for this observation is that patients required to take a larger number of oral AEDs may have had more severe forms of focal epilepsy. ${ }^{41}$

We are willing to share our ECoG dataset with investigators who express an interest in testing the performance of MI or other measures of their interest (eg, occurrence rate of interictal HFA). Our study was not designed to determine whether $\mathrm{MI}_{(>150 \mathrm{~Hz}}$ \& 3-4 Hz) is more useful than other interictal HFA measures. Some investigators have suggested that characterization of the angle of slow-wave phase preferentially coupled with HFA was useful in localization of SOZ. ${ }^{42-44}$ For measurement of the amplitude of HFA, the present study employed a high-pass filter of $150 \mathrm{~Hz}$; thus, the amplitude of $\mathrm{HFA}_{80-150 \mathrm{~Hz}}$ may have only modestly contributed to computation of $\mathrm{MI}_{(>150 \mathrm{~Hz} \& 3-4 \mathrm{~Hz})}$. HFA $80-150 \mathrm{~Hz}$ 
and $\mathrm{HFA}_{>150 \mathrm{~Hz}}$ are also known as ripples and fast ripples, respectively. ${ }^{29,45}$ Several studies demonstrated that $\mathrm{HFA}_{>80 \mathrm{~Hz}}, \mathrm{HFA}_{>150 \mathrm{~Hz}}$, and $\mathrm{HFA}_{>250 \mathrm{~Hz}}$ largely share similar spatial-temporal profiles. ${ }^{19,35}$ Previous ECoG studies reported that regions showing interictal spike discharges associated with increased $\mathrm{HFA}_{>80 \mathrm{~Hz}}$ amplitude or $\mathrm{HFA}_{>80 \mathrm{~Hz}}$ coupled with slow waves $3-4 \mathrm{~Hz}$ often turned out to involve the SOZ, ${ }^{19,21,33-35,46}$ whereas high-amplitude, sharply contoured transients at alpha/beta frequency range can be observed in nonepileptic regions. ${ }^{47,48}$

We speculate that MI during slow-wave sleep and stage 2 sleep would yield a similar clinical utility in epilepsy presurgical evaluation. These sleep stages share a similar MI spatial pattern (Figure 4). The mixed model analysis demonstrated that the contrast on MI between SOZ and non-SOZ was about 10 times greater than that between non-REM sleep and the other stages (estimate of $\mathrm{SOZ}=$ +0.045 vs estimate of non-REM sleep $=+0.004$ ).

This is a retrospective observational study, which should be considered in the interpretation of our results. Our results by no means indicate that cortical areas showing increased MI should be blindly removed to optimize the postoperative outcome. Epilepsy surgery teams should estimate the extent of the epileptogenic zone by taking into account the clinical context of a given patient. A multicenter prospective study is warranted to determine how universally useful the MI variable is in presurgical evaluation. It remains unknown how the utility of MI is altered by different electrode types (eg, disk vs depth electrodes), different montage (eg, common average reference vs bipolar montage ${ }^{27}$ ), anesthesia conditions, and intracranial electrode placement approaches (eg, large vs limited coverage). A retrospective study of 54 patients reported that the occurrence of $\mathrm{HFA}_{>250 \mathrm{~Hz}}$ on intraoperative ECoG immediately following cortical resection was associated with a greater risk of seizure recurrence. ${ }^{15}$ One logical next step would be to determine whether quantitative measurement of MI during intraoperative ECoG recording will provide useful information to localize the EZ.

\section{ACKNOWLEDGMENT}

This work was supported by National Institutes of Health grants NS047550 (to E.A.), NS064033 (to E.A.), and NS089659 (to J.W.J.). We are grateful to Harry T. Chugani, Aashit Shah, Sandeep Mittal, and Deniz Altinok at Children's Hospital of Michigan, Wayne State University, Detroit Medical Center for the collaboration and assistance in performing the studies described above.

\section{DISCLOSURE}

The authors have no conflicts of interest to report. We confirm that we have read the Journal's position on issues involved in ethical publication and affirm that this report is consistent with those guidelines.

\section{OR CID}

Eishi Asano (iD http://orcid.org/0000-0001-8391-4067

\section{REFERENCES}

1. Rosenow F, Lüders H. Presurgical evaluation of epilepsy. Brain. 2001;124:1683-700.

2. Jehi L. the epileptogenic zone: concept and definition. Epilepsy Curr. 2018;18:12-6.

3. Bartolomei F, Chauvel P, Wendling F. Epileptogenicity of brain structures in human temporal lobe epilepsy: a quantified study from intracerebral EEG. Brain. 2008;131:1818-30.

4. Fujiwara H, Greiner HM, Lee $\mathrm{KH}$, et al. Resection of ictal highfrequency oscillations leads to favorable surgical outcome in pediatric epilepsy. Epilepsia. 2012;53:1607-17.

5. Korzeniewska A, Cervenka MC, Jouny $\mathrm{CC}$, et al. Ictal propagation of high frequency activity is recapitulated in interictal recordings: effective connectivity of epileptogenic networks recorded with intracranial EEG. Neuroimage. 2014;101:96-113.

6. Weiss SA, Lemesiou A, Connors R, et al. Seizure localization using ictal phase-locked high gamma: a retrospective surgical outcome study. Neurology. 2015;84:2320-8.

7. Kahane P, Landré E, Minotti L, et al. The Bancaud and Talairach view on the epileptogenic zone: a working hypothesis. Epileptic Disord. 2006;8(suppl 2):16-26.

8. Asano E, Juhász C, Shah A, et al. Role of subdural electrocorticography in prediction of long-term seizure outcome in epilepsy surgery. Brain. 2009;132:1038-47.

9. Jacobs J, Zijlmans M, Zelmann R, et al. High-frequency electroencephalographic oscillations correlate with outcome of epilepsy surgery. Ann Neurol. 2010;67:209-20.

10. Akiyama T, McCoy B, Go CY, et al. Focal resection of fast ripples on extraoperative intracranial EEG improves seizure outcome in pediatric epilepsy. Epilepsia. 2011;52:1802-11.

11. Usui N, Terada K, Baba K, et al. Significance of very-high-frequency oscillations (over 1,000 Hz) in epilepsy. Ann Neurol. 2015;78:295-302.

12. Brázdil M, Pail M, Halámek J, et al. Very high-frequency oscillations: novel biomarkers of the epileptogenic zone. Ann Neurol. 2017;82:299-310.

13. Fedele T, Burnos S, Boran E, et al. Resection of high frequency oscillations predicts seizure outcome in the individual patient. Sci Rep. 2017;7:13836.

14. Hussain SA, Mathern GW, Hung P, et al. Intraoperative fast ripples independently predict postsurgical epilepsy outcome: comparison with other electrocorticographic phenomena. Epilepsy Res. 2017;135:79-86.

15. van't Klooster MA, van Klink NEC, Zweiphenning WJEM, et al. Tailoring epilepsy surgery with fast ripples in the intraoperative electrocorticogram. Ann Neurol. 2017;81:664-76.

16. Höller Y, Kutil R, Klaffenböck L, et al. High-frequency oscillations in epilepsy and surgical outcome. A meta-analysis. Front Hum Neurosci. 2015;9:574. 
17. Canolty RT, Edwards E, Dalal SS, et al. High gamma power is phase-locked to theta oscillations in human neocortex. Science. 2006;313:1626-8.

18. Miyakoshi M, Delorme A, Mullen T, et al. Automated detection of cross-frequency coupling in the electrocorticogram for clinical inspection. Conf Proc IEEE Eng Med Biol Soc. 2013;2013:3282-5.

19. Nonoda Y, Miyakoshi M, Ojeda A, et al. Interictal high-frequency oscillations generated by seizure onset and eloquent areas may be differentially coupled with different slow waves. Clin Neurophysiol. 2016;127:2489-99.

20. Iimura Y, Jones K, Hattori K, et al. Epileptogenic high-frequency oscillations skip the motor area in children with multilobar drugresistant epilepsy. Clin Neurophysiol. 2017;128:1197-205.

21. Iimura Y, Jones K, Takada L, et al. Strong coupling between slow oscillations and wide fast ripples in children with epileptic spasms: investigation of modulation index and occurrence rate. Epilepsia. 2018;59:544-54.

22. Frauscher B, von Ellenrieder N, Zelmann R, et al. Atlas of the normal intracranial electroencephalogram: neurophysiological awake activity in different cortical areas. Brain. 2018;141:1130-44.

23. Miller KJ, Hermes D, Honey CJ, et al. Dynamic modulation of local population activity by rhythm phase in human occipital cortex during a visual search task. Front Hum Neurosci. 2010;4:197.

24. Asano E, Chugani DC, Juhász C, et al. Surgical treatment of West syndrome. Brain Dev. 2001;23:668-76.

25. Nakai Y, Jeong JW, Brown EC, et al. Three- and four-dimensional mapping of speech and language in patients with epilepsy. Brain. 2017;140:1351-70.

26. Ghosh SS, Kakunoori S, Augustinack J, et al. Evaluating the validity of volume-based and surface-based brain image registration for developmental cognitive neuroscience studies in children 4 to 11 years of age. Neuroimage. 2010;53:85-93.

27. Kambara T, Sood S, Alqatan Z, et al. Presurgical language mapping using event-related high-gamma activity: the Detroit procedure. Clin Neurophysiol. 2018;129:145-54.

28. Nariai H, Nagasawa T, Juhász C, et al. Statistical mapping of ictal high-frequency oscillations in epileptic spasms. Epilepsia. 2011;52:63-74.

29. Nagasawa T, Juhász C, Rothermel R, et al. Spontaneous and visually driven high-frequency oscillations in the occipital cortex: intracranial recording in epileptic patients. Hum Brain Mapp. 2012;33:569-83.

30. Bagshaw AP, Jacobs J, LeVan P, et al. Effect of sleep stage on interictal high-frequency oscillations recorded from depth macroelectrodes in patients with focal epilepsy. Epilepsia. 2009;50:617-28.

31. Bénar CG, Chauvière L, Bartolomei F, et al. Pitfalls of high-pass filtering for detecting epileptic oscillations: a technical note on "false" ripples. Clin Neurophysiol. 2010;121:301-10.

32. Shamas M, Benquet P, Merlet I, et al. On the origin of epileptic high frequency oscillations observed on clinical electrodes. Clin Neurophysiol. 2018;129:829-41.

33. Burnos S, Frauscher B, Zelmann R, et al. The morphology of high frequency oscillations (HFO) does not improve delineating the epileptogenic zone. Clin Neurophysiol. 2016;127:2140-8.

34. Roehri N, Pizzo F, Lagarde S, et al. High-frequency oscillations are not better biomarkers of epileptogenic tissues than spikes. Ann Neurol. 2018;83:84-97.

35. Jacobs J, Kobayashi K, Gotman J. High-frequency changes during interictal spikes detected by time-frequency analysis. Clin Neurophysiol. 2011;122:32-42.
36. Wieser HG, Blume WT, Fish D, et al. Commission on Neurosurgery of the International League Against Epilepsy (ILAE). ILAE Commission Report. Proposal for a new classification of outcome with respect to epileptic seizures following epilepsy surgery. Epilepsia. 2001;42:282-6.

37. Nakai Y, Nagashima A, Hayakawa A, et al. Four-dimensional map of the human early visual system. Clin Neurophysiol. 2018;129:188-97.

38. Steriade M, Timofeev I. Neuronal plasticity in thalamocortical networks during sleep and waking oscillations. Neuron. 2003;37:563-76.

39. Téllez-Zenteno JF, Hernández Ronquillo L, Moien-Afshari F, et al. Surgical outcomes in lesional and non-lesional epilepsy: a systematic review and meta-analysis. Epilepsy Res. 2010;89:310-8.

40. Englot DJ, Breshears JD, Sun PP, et al. Seizure outcomes after resective surgery for extra-temporal lobe epilepsy in pediatric patients. J Neurosurg Pediatr. 2013;12:126-33.

41. Kwan P, Brodie MJ. Neuropsychological effects of epilepsy and antiepileptic drugs. Lancet. 2001;357:216-22.

42. Frauscher B, von Ellenrieder N, Ferrari-Marinho T, et al. Facilitation of epileptic activity during sleep is mediated by high amplitude slow waves. Brain. 2015;138:1629-41.

43. von Ellenrieder N, Frauscher B, Dubeau F, et al. Interaction with slow waves during sleep improves discrimination of physiologic and pathologic high-frequency oscillations $(80-500 \mathrm{~Hz})$. Epilepsia. 2016;57:869-78.

44. Song I, Orosz I, Chervoneva I, et al. Bimodal coupling of ripples and slower oscillations during sleep in patients with focal epilepsy. Epilepsia. 2017;58:1972-84.

45. Ogren JA, Wilson CL, Bragin A, et al. Three-dimensional surface maps link local atrophy and fast ripples in human epileptic hippocampus. Ann Neurol. 2009;66:783-91.

46. Wang S, So NK, Jin B, et al. Interictal ripples nested in epileptiform discharge help to identify the epileptogenic zone in neocortical epilepsy. Clin Neurophysiol. 2017;128:945-51.

47. Mizrahi EM. Avoiding the pitfalls of EEG interpretation in childhood epilepsy. Epilepsia. 1996;37(suppl 1):S41-51.

48. Sperling MR. Intracranial electroencephalography. In: Ebersole JS, Pedley TA, eds. Current Practice of Clinical Electroencephalography. New York, NY: Lippincott Williams and Wilkins; 2003:639-80.

\section{SUPPORTING INFORMATION}

Additional supporting information may be found online in the Supporting Information section at the end of the article.

How to cite this article: Motoi H, Miyakoshi M, Abel TJ, et al. Phase-amplitude coupling between interictal high-frequency activity and slow waves in epilepsy surgery. Epilepsia. 2018;59:1954-1965. https://doi.org/10.1111/epi.14544 